\title{
ARTICLE Stiffening of sickle cell trait red blood cells under simulated strenuous exercise conditions
}

\author{
Zhensong $\mathrm{Xu}^{1}$, Yi Zheng ${ }^{1,2}$, Xian Wang ${ }^{1}$, Nadine Shehata ${ }^{3}$, Chen Wang ${ }^{4,5}$, Shaorong Xie ${ }^{6}$ and Yu Sun ${ }^{1,2,6,7}$
}

The higher risk of vaso-occlusion events and sudden death for sickle-cell trait (SCT) athletes has been speculatively ascribed to SCT red blood cell (RBC) stiffening during strenuous exercise. However, the microenvironmental changes that could induce the stiffening of SCT RBCs are unknown. To address this question, we measured the mechanical properties of and changes in SCT RBCs under deoxygenated and acidic environments, which are two typical conditions present in the circulation of athletes undertaking strenuous exercise. The results reveal that SCT RBCs are inherently stiffer than RBCs from non-SCT healthy subjects, and a lower pH further stiffens the SCT cells. Furthermore, at both normal and low pH levels, deoxygenation was found to not be the cause of the stiffness of SCT RBCs. This study confirms that the stiffening of SCT RBCs occurs at a low pH and implies that SCT RBC stiffening could be responsible for vaso-occlusion in SCT athletes during strenuous exercise.

Keywords: deformability; microfluidics; shear modulus; sickle cell trait; stiffening; red blood cell

Microsystems \& Nanoengineering (2016) 2, 16061; doi:10.1038/micronano.2016.61; Published online: 7 November 2016

\section{INTRODUCTION}

Athletes are often considered the healthiest members of the human population; hence, the occurrence of sudden deaths in this group ${ }^{1}$ can be shocking and has a devastating impact on communities and the public ${ }^{2,3}$. According to an analysis conducted by the National Collegiate Athletic Association (NCAA) from 2004 to 2008 (Ref. 1), 36 out of 80 medical causes of athletes' death $(45 \%)$ were identified to be exertional sudden deaths ${ }^{4}$. Among all exertional sudden deaths, sickle cell trait (SCT)-related cases caused the most controversies ${ }^{5-7}$.

SCT describes the inheritance of one normal hemoglobin gene $(\mathrm{HbA})$ from one parent along with one mutated $\beta 1$-globin gene, the sickle hemoglobin gene $(\mathrm{HbS})$, from the other parent ${ }^{8}$. At the end of 2009, there were 300 million people worldwide with SCT.

SCT is typically considered to be benign and harmless ${ }^{9}$. However, as more cases of SCT athletes' sudden deaths were reported ${ }^{4,10}$, heated debates over whether SCT should be considered as a cause of death during exercise and whether athletes should be screened for SCT arose. Rationally, analyzing these issues requires a clear understanding of the properties of SCT RBCs ${ }^{4,11,12}$.

Vaso-occlusion, usually caused by the blockage of blood vessels, is one of the most fatal and common symptoms of sickle cell disease (SCD). It also appears to be a crucial contributor to sudden deaths in SCT individuals ${ }^{12}$. RBCs from SCD patients are known to become stiffer and even sickled under deoxygenated or acidic conditions, causing the blockage of blood vessels ${ }^{13,14}$. However, whether SCT RBCs also become stiffer under deoxygenated or acidic conditions is not known.

During strenuous exercise, athletes' muscles are under a maximal oxygen consuming condition, which lowers the oxygen level in the circulation. Furthermore, although human blood $\mathrm{pH}$ is normally 7.35, in strenuous exercise, a higher concentration of hydrogen ions in the human body causes blood $\mathrm{pH}$ to drop below 7.0 and even to 6.8 under extreme conditions for a short time period $^{15,16}$.

In this study, we focused on determining whether lowered $\mathrm{pH}$ and deoxygenation conditions can trigger the stiffening of SCT RBCs, which could be associated with higher vaso-occlusion risks. Measurements were taken on both normal RBCs and SCT RBCs using a microfluidic system that is capable of controlling oxygen and $\mathrm{pH}$ levels.

\section{MATERIALS AND METHODS Blood specimens}

The study was performed in accordance with the institutional guidelines for using human tissue samples. Blood samples were collected for routine tests and used for study only after they were completed for clinical tests and would be otherwise discarded. The study protocol was approved by the Mount Sinai Hospital Research Ethics Board, in which informed consent was not required because the samples were selected retrospectively, no patient identification was disclosed to the study, and the study had no effect on the clinical test or patient management. SCT was confirmed by sickle cell testing and standard hemoglobin electrophoresis in the clinical laboratory. Blood samples, including seven normal blood samples and seven SCT blood samples, were stored with ethylenediaminetetraacetic acid (EDTA, $1.5 \mathrm{mg} \mathrm{ml}^{-1}$ ) and used within $48 \mathrm{~h}$. Before they were introduced into the device under room temperature,

\footnotetext{
${ }^{1}$ Department of Mechanical and Industrial Engineering, University of Toronto, Toronto, ON, Canada; ${ }^{2}$ Institute of Biomaterials and Biomedical Engineering, University of Toronto, Toronto, ON, Canada; ${ }^{3}$ Department of Medicine, University of Toronto, Toronto, ON, Canada; ${ }^{4}$ Department of Pathology and Laboratory Medicine, Mount Sinai Hospital, Toronto, ON, Canada; ${ }^{5}$ Department of Laboratory Medicine and Pathobiology, University of Toronto, Toronto, ON, Canada; ${ }^{6}$ School of Mechatronic Engineering and Automation, Shanghai University, Shanghai, China and ${ }^{7}$ Department of Electrical and Computer Engineering, University of Toronto, Toronto, ON, Canada.

Correspondence: Chen Wang (cwang@mtsinai.on.ca) or Shaorong Xie (srxie@shu.edu.cn) or Yu Sun (sun@mie.utoronto.ca)

Received: 15 May 2016; revised: 21 July 2016; accepted: 21 July 2016
} 


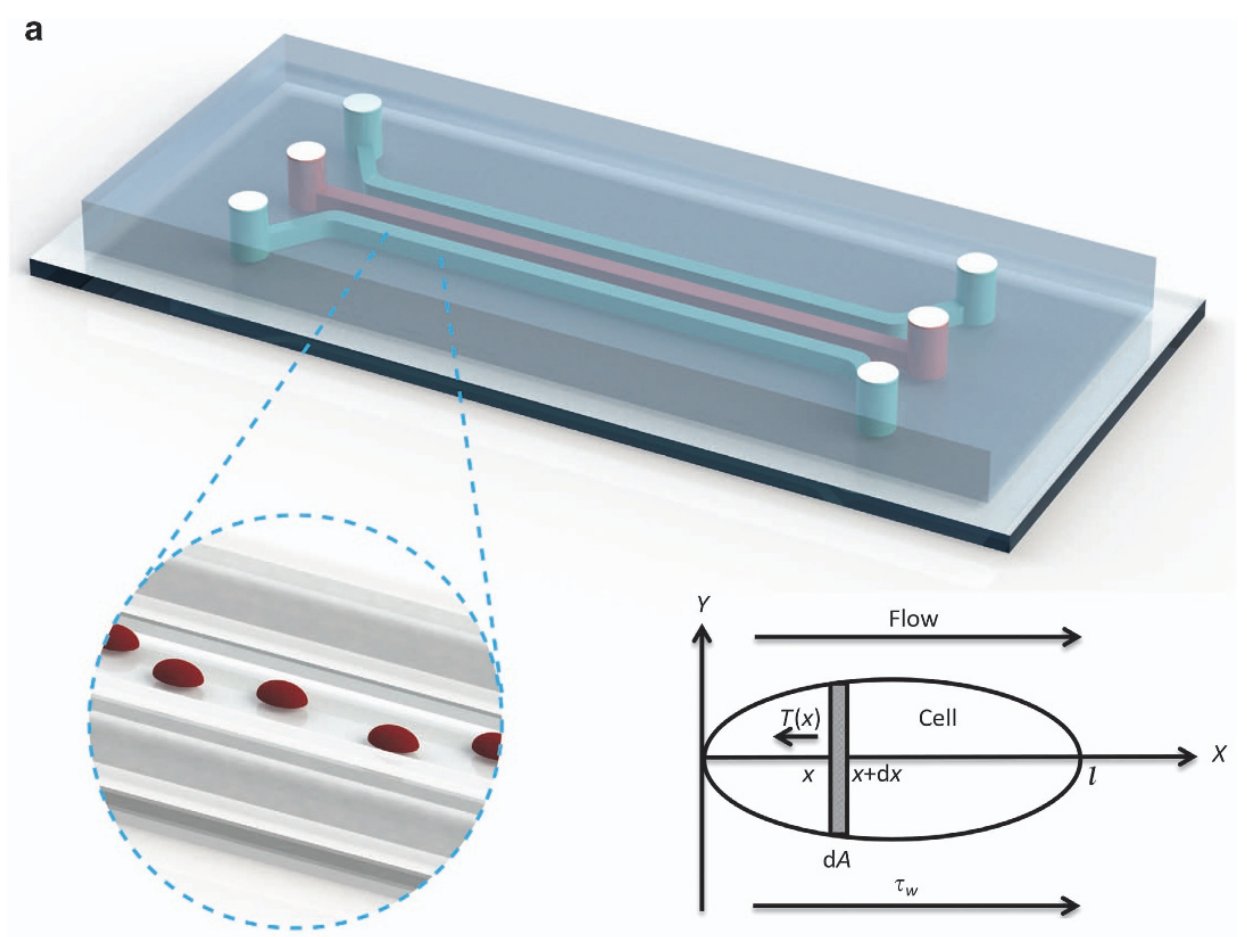

b Shear applied
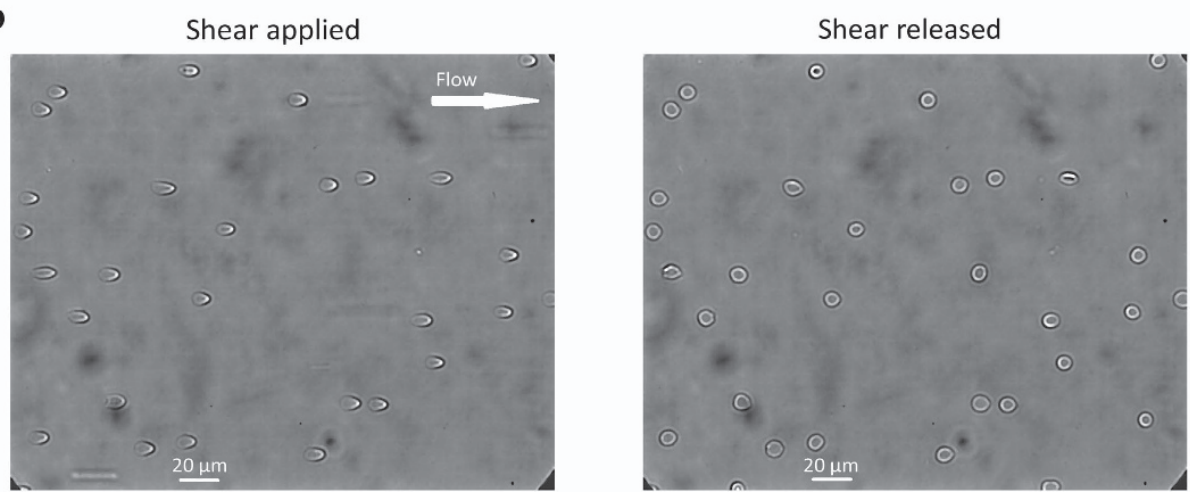

Figure 1 (a) Schematic of the microfluidic device used for RBC mechanical property measurement. RBCs in solutions of different pH levels are loaded into the middle channel and adhere onto the glass channel bottom. The oxygen level is accurately varied by pumping air or nitrogen into the two side channels. Schematic illustration of the deformation of a cell element. (b) RBCs are deformed under shear stress (1.8 kPa). After the release of shear stress, RBCs recover to their original shape.

blood samples tested under normal $\mathrm{pH}$ level were diluted 200 times in phosphate-buffered saline (PBS, pH=7.35 \pm 0.05 ), whereas blood samples tested under acidic conditions were diluted 200 times in acid-adjusted PBS $(\mathrm{pH}=7.10 \pm 0.05$ and $\mathrm{pH}=6.85 \pm 0.05)$.

\section{Device and measurement}

As shown in Figure 1a, the microfluidic device consists of three parallel channels. The middle channel is used for loading and testing RBCs, and the other two channels are used to control the oxygen level in the middle channel. After a diluted blood sample is introduced into the middle channel, RBCs strongly adhere to the glass substrate due to the difference in electrical charge on the cell membrane and glass surface ${ }^{17}$. In experiments, when the pressure was varied from $0 \mathrm{~Pa}$ to $9 \mathrm{kPa}$, none of the RBCs were detached or revealed noticeable displacements. Two water tanks containing PBS are connected to the inlet and outlet of the middle channel to maintain the osmolality and $\mathrm{pH}$ level. Pumping either air or nitrogen into the two side channels controls the oxygen level in the device owing to the gas permeability characteristic of polydimethylsiloxane $(\mathrm{PDMS})^{18}$. The crosssectional area of the three channels is $60 \mu \mathrm{m} \times 300 \mu \mathrm{m}$. The gap between neighboring channels is $100 \mu \mathrm{m}$ for facilitated gas exchange between the gas channel and the cell testing channel. Details of fabrication and design of the microfluidic device have been described in our previous study ${ }^{19}$. In addition, the $\mathrm{pH}$ level is controlled by adding hydrochloric acid and confirmed before and after each experiment by using a $\mathrm{pH}$ measurement instrument (Hanna FC 240B pH electrode). RBCs are deformed under shear stress $(0.9 \mathrm{~Pa})$ generated by a regulated vacuum source (pressure difference is $1.8 \mathrm{kPa}$ ), and this shear stress is comparable to the in vivo condition ${ }^{20}$. After the release of the shear stress, the RBCs recover to their original shapes. The dynamic recovery process is captured using a CCD camera connected to a microscope. Mechanical models are developed to extract the shear modulus of each RBC. 


\section{RESULTS}

\section{Determination of RBC shear modulus}

When an RBC is in a shear flow, its membrane undergoes deformation by shear stress. According to the Kelvin-Voigt (KV) model $^{21,22}$

$$
\bar{T}=\frac{\mu}{2}\left(\lambda^{2}-\frac{1}{\lambda^{2}}\right)
$$

where $\bar{T}\left(\mu \mathrm{N} \mathrm{m}^{-1}\right)$ is the average tension force acting on the RBC membrane, $\mu\left(\mu \mathrm{N} \mathrm{m}^{-1}\right)$ is the elastic shear modulus expressed in force per unit length, and $\lambda$ is the extension ratio of the RBC membrane. $\lambda=\frac{l}{I_{0}}$, where $I$ is the RBC's length when deformed under shear stress, and $I_{0}$ is the RBC's original length.

The flow in the microfluidic channel in this study is driven by a pressure difference $(\Delta P)$. The velocity profile of pressure-driven flow is

$$
\nu=\frac{\pi R^{2} \Delta P}{4 \eta L}\left(1-\frac{r^{2}}{R^{2}}\right)
$$

where $\eta$ is the dynamic viscosity of the fluid and $L$ is the length of the channel. Because the microchannel has a rectangular crosssection and its width $(w)$ is much larger than the channel height (h), hydraulic radius $R$ is approximately equal to $h$. Because $w \gg h$, the error caused by the approximation is minor and does not affect the result in this paper. Shear stress on the microchannel bottom where RBCs are located is

$$
\tau_{w}=\left.\eta \frac{\mathrm{d} \nu}{\mathrm{d} r}\right|_{r=R}=\frac{\Delta P h}{2 L}
$$

To calculate tension force $\bar{T}$ in the direction of extension, a small element $(\mathrm{d} A)$ is taken for force equilibrium analysis, as shown in Figure 1, and shear flow only applies in the $x$ direction. Because only steady-state behavior is considered, the tension force and shear force on each element are balanced ${ }^{21,23}$.

$$
2 \mathrm{~d}\left[T_{x} Y(x)\right]=\tau_{w} \mathrm{~d} A
$$

where $Y(x)$ is the half-width of the element $\mathrm{d} A$. The average tension force $\bar{T}$ is ${ }^{24}$

$$
\bar{T}=\frac{\int_{0}^{l} T(x) \mathrm{d} x}{l} \approx \frac{\tau_{w} A}{l_{\mathrm{b}}}
$$

where $A$ is the surface area of the RBC, and $I_{b}$ is the width of the RBC. Shear modulus $\mu$ is determined by substituting Equation (5) into Equation (1).

\section{SCT RBCs become stiffened under lower pH}

RBCs from healthy donors (control) and SCT individuals were first tested using the microsystem without adjusting the $\mathrm{pH}$ level. Figure 2 shows that under normal pH levels, SCT RBCs are significantly stiffer than normal RBCs, which agrees with previous results ${ }^{19,25}$. The higher stiffness of SCT RBCs could lead to a higher blood viscosity in the vascular system ${ }^{26}$. In large blood vessels, lower RBC deformability limits cell orientation in flow and thus increases blood viscosity ${ }^{27}$. In small blood vessels, stiffer RBCs lead to a lower Fahraeus-Lindqvist effect, which increases the flow resistance and blood viscosity ${ }^{28}$. In healthy non-SCT carriers, endothelial cells lining the blood vessels can generate vasodilators (e.g., nitric oxide) to mediate increased blood viscosity. In contrast, SCT carriers are known to develop impaired vascular functions and generate fewer vasodilators ${ }^{12,29}$.

We next measured the shear modulus of both SCT and normal RBCs at lower pH levels. As shown in Figure $2 \mathrm{a}$, the shear modulus of all seven tested SCT RBC samples consistently increased under a low $\mathrm{pH}$ level of $6.85(P<0.05)$. This low $\mathrm{pH}$ level was chosen because it is known that in strenuous exercise, a higher concentration of hydrogen ions in the human body can cause the $\mathrm{pH}$ level to drop to approximately 6.8 (Refs. 15,16). We then aimed to investigate whether moderate exercise could induce SCT RBC stiffening. Hence, we conducted experiments under an intermediate $\mathrm{pH}$ value $(\mathrm{pH}$ 7.10) that mimics the moderate exercise condition, and no significant difference in shear modulus was observed, as shown in Figure 3c.

In contrast, RBCs from normal subjects did not respond significantly to low $\mathrm{pH}$ levels. When $\mathrm{pH}$ was reduced to 6.85 , as shown in Figure $2 \mathrm{~b}$ including seven samples' data, the shear modulus of normal RBCs increased slightly. However, the difference was not statistically significant $(P \geqslant 0.05)$. In our experiments, normal RBCs started to reveal stiffness changes with statistical significance only at $\mathrm{pH}$ levels $<6.0$ (data not shown), which is a physiologically irrelevant condition ${ }^{30-32}$.

As shown in Figure $2 \mathrm{c}$ in which all seven samples are summarized together and reported in boxplots, when $\mathrm{pH}$ was reduced from 7.35 to 6.85 , SCT RBCs were stiffened significantly, whereas control RBCs' stiffness increased only slightly. The average shear modulus of normal RBCs increased from $2.09 \pm 0.67$ to $2.27 \pm 0.83 \mu \mathrm{N} \mathrm{m}^{-1}$, whereas the average shear modulus of RBCs from SCT individuals increased significantly from $2.81 \pm 0.7$ to $4.05 \pm 1.08 \mu \mathrm{N} \mathrm{m}^{-1}$. The results indicate that SCT RBCs are inherently stiffer than control/normal RBCs, and SCT RBCs are more sensitive to lowered $\mathrm{pH}$ levels than normal RBCs. Statistical analysis confirms a significant difference of the shear moduli of normal RBCs and SCT RBCs under the acidic condition ( $2.27 \pm 0.83$ vs. $\left.4.05 \pm 1.08 \mu \mathrm{N} \mathrm{m}^{-1} ; P=1.9 \times 10^{-82}\right)$. The significant stiffening of SCT RBCs under the acidic condition could cause difficulties for the SCT RBCs to pass through minuscule vessels and capillaries. Along with the increased blood viscosity, this could lead to a higher chance of vessel blockage. The resulting vaso-occlusion events could result in acute ventricular failure ${ }^{33-35}$, which could contribute to sudden death in SCT carriers.

\section{Hypoxia does not induce SCT RBC stiffening}

We then tested the oxygen effect on SCT and normal RBCs. As shown in Figure 3, the stiffness of SCT RBCs was not found to increase after deoxygenation under physiological pH (7.35), which is in agreement with the previously reported result ${ }^{19}$. We speculated that under acidic conditions, deoxygenation might cause SCT RBCs to become even stiffer compared with the condition of low $\mathrm{pH}$ only. However, the measurement results revealed that deoxygenation is not capable of stiffening SCT RBCs further. In the deoxygenation experiments, we infused nitrogen into the two side channels for $20 \mathrm{~min}$ to reduce the oxygen concentration from $20 \%$ (air) to $0 \%$ (pure nitrogen). The validation of oxygen depletion was described in our previous study ${ }^{19}$.

As discussed in the previous section, the shear modulus of SCT RBCs increased from $2.81 \pm 0.7$ to $4.05 \pm 1.08 \mu \mathrm{N} \mathrm{m}^{-1}$ under $\mathrm{pH} 7.35$ and 6.85. These shear modulus values largely remained the same when the channel was deoxygenated (for $\mathrm{pH} 7.35$, oxygenated: $2.81 \pm 0.7 \mu \mathrm{N} \mathrm{m}^{-1}$ vs. deoxygenated: $2.82 \pm 0.7 \mu \mathrm{N} \mathrm{m}^{-1}$; for $\mathrm{pH} 6.85$, oxygenated: $4.05 \pm 1.08 \mu \mathrm{N} \mathrm{m}^{-1}$ vs. deoxygenated: $\left.4.02 \pm 1.04 \mu \mathrm{N} \mathrm{m}^{-1}\right)$. The differences were confirmed by using Mann-Whitney nonparametric analysis to be insignificant $(P>0.75)$. To decouple the effect of deoxygenation and $\mathrm{pH}$ levels, the RBC samples were diluted 200 times, and it was confirmed after each experiment that there was no significant difference in $\mathrm{pH}$ after deoxygenation.

\section{DISCUSSION}

SCT was recently reported to be associated with strenuous exercise-related mortality ${ }^{4,5,10}$. Existing results on the mechanical properties of RBCs from SCT individuals are limited. Here we examined SCT RBCs' stiffness change under controlled oxygen and $\mathrm{pH}$ levels. The results reveal that SCT RBCs are significantly stiffer 


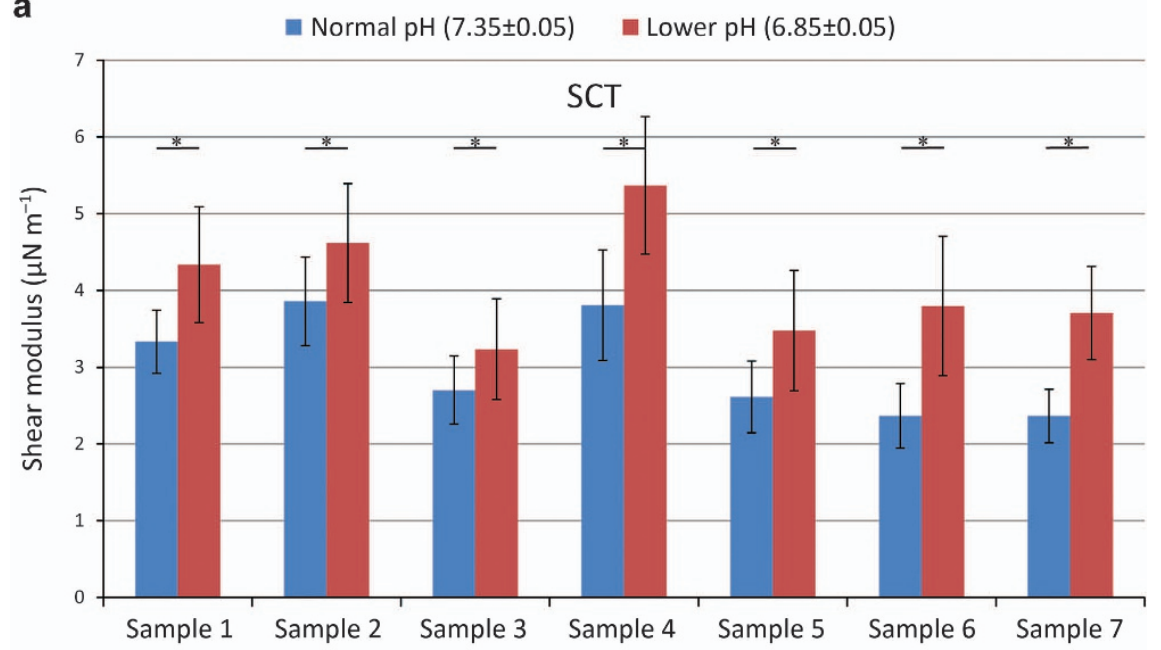

b

normal pH (7.35 \pm 0.05$) \quad$ a Lower $\mathrm{pH}(6.85 \pm 0.05)$

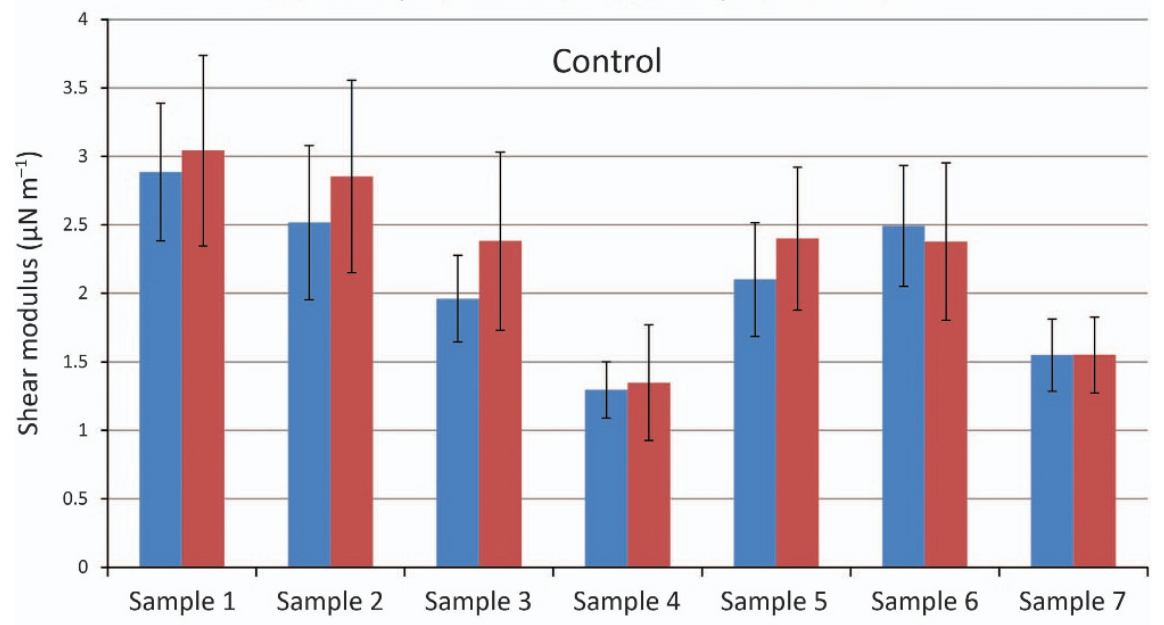

C

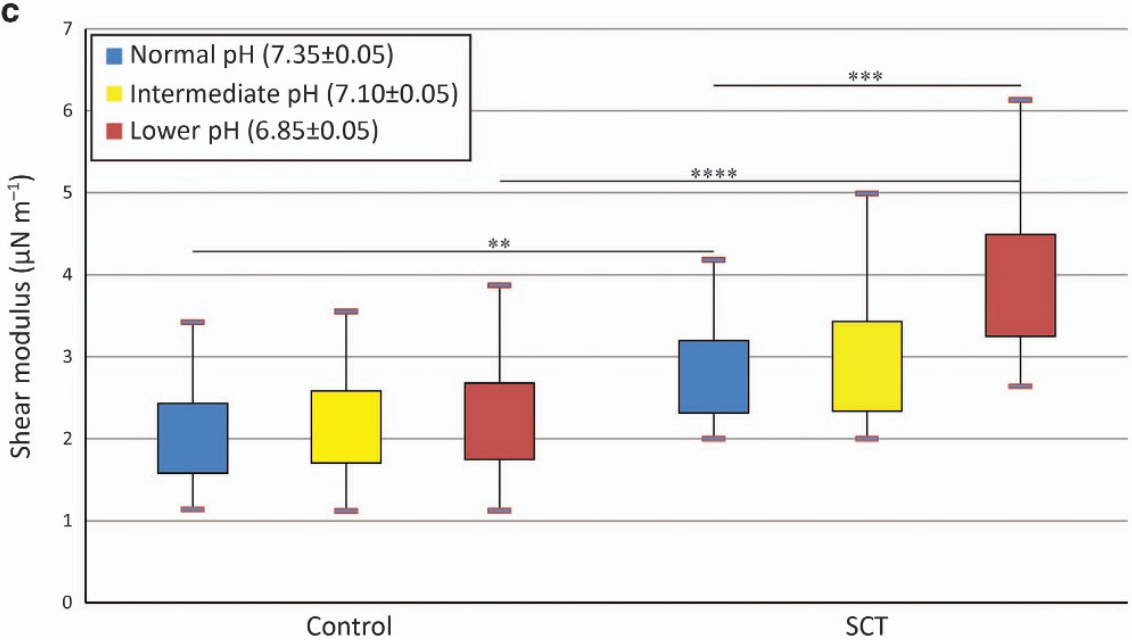

Figure 2 (a) Shear modulus of SCT RBCs under a physiological pH level of 7.35 (blue) and an acidic pH level of 6.85 (red). Error bars represent the standard deviation. All samples $\left(n=30-70\right.$ for each sample) show a significant difference between different pH levels $\left({ }^{*} P<0.05\right)$. (b) The shear modulus of normal RBCs did not reveal a significant difference under different pH levels $(P$ value $>0.05 ; n=30-70$ for each sample). Error bars represent the standard deviation. (c) Boxplot showing the summarized shear modulus of RBCs from the seven tested SCT samples and the seven tested normal samples under different $\mathrm{pH}$ conditions $\left({ }^{* *} P=5.5 \times 10^{-15},{ }^{* * *} P=2.2 \times 10^{-29},{ }^{* * * *} P=1.9 \times 10^{-82}\right.$; $n=200-300$ ). 

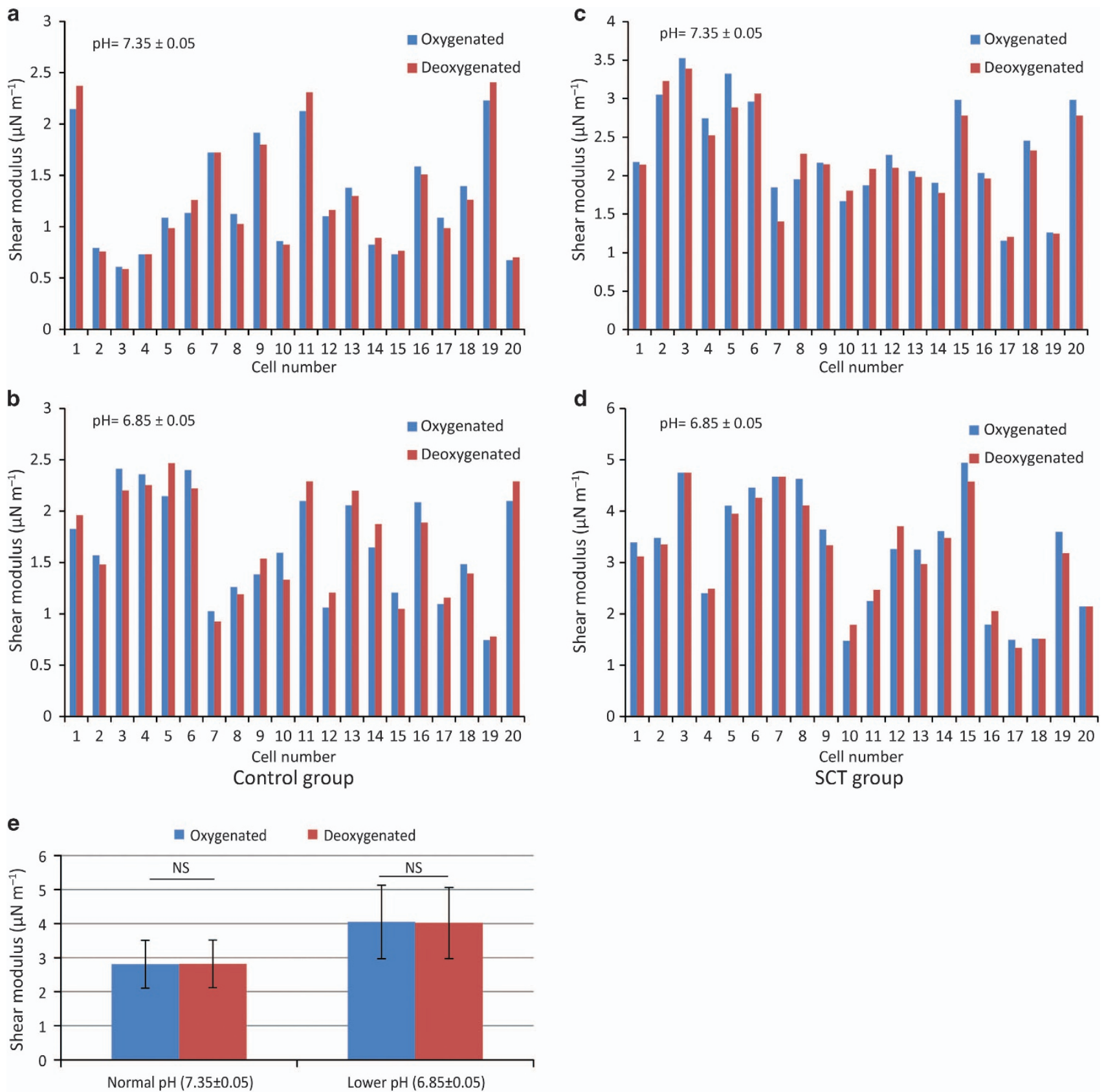

Figure 3 At physiological pH 7.35 (a) and low pH 6.85 (b), the normal RBCs' elastic modulus did not change significantly when the channel was deoxygenated $\left({ }^{*} P>0.75\right.$ ). (c and d) For SCT RBCs, although the shear modulus increased at pH 6.85 compared with pH 7.35, deoxygenation did not induce a further increase $\left({ }^{*} P>0.75\right)$. (e) Summarized shear modulus of SCT RBCs under different oxygen levels, in which no difference was observed. Error bars represent the standard deviation.

than RBCs from non-SCT healthy subjects. A lower $\mathrm{pH}$ resulted in a $28 \%$ increase in SCT RBCs' shear modulus (Figure 2).

The stiffness increase of SCT RBCs could be due to several physiological alterations. It was reported that SCT RBCs contain a higher concentration of $\mathrm{Ca}^{+2}$, which can enhance the binding of the cytoplasmic domain of band 3 (CDB3) to the cytoskeleton-bound Ankyrin $^{25,36}$. This stronger binding caused by increased $\mathrm{Ca}^{+2}$ can possibly contribute to the higher rigidity of the membrane of SCT RBCs. At lower $\mathrm{pH}, \mathrm{CDB} 3$ becomes even more compact ${ }^{30,37}$, which can further enhance the binding and stiffening of SCT RBCs. In addition, monocarboxylate transporter 1 (MCT-1) activity has been speculated to impact RBC stiffness ${ }^{38-40}$. Because MCT-1 activity is inherently stronger in SCT RBCs than normal RBCs at low pH, the higher concentration of hydrogen ions leads to even stronger MCT-1 activity, and the enhancement of MCT-1 activity could also be responsible for the significant increase in shear modulus measured on SCT RBCs at pH 6.85. However, because no significant difference was observed when $\mathrm{pH}$ was lowered to 7.10, we speculate that at $\mathrm{pH} 7.10$, the cytoplasmic domain of band 3 (CDB3) was not sufficiently compact to enhance the binding to the cytoskeleton-bound Ankyrin, leading to insignificant stiffening of RBCs. The results also suggest that under normal exercise conditions in which $\mathrm{pH}$ only slightly decreases, the stiffening of SCT RBCs would not be significantly evident.

The experimental results also show that deoxygenation did not induce further stiffening of SCT RBCs (Figure 3). During strenuous 
exercise, both blood oxygen and $\mathrm{pH}$ levels decreased. Although deoxygenation did not directly impact the stiffness of SCT RBCs, low oxygen levels during exercise can contribute to the lowering of blood $\mathrm{pH}^{41}$. Our data measured in the simulated strenuous exercise condition indicate that low $\mathrm{pH}$ rather than hypoxia is effective in triggering SCT RBC stiffening. In addition, although SCD RBCs are known to sickle under acidic and/or hypoxia conditions, no sickling of SCT RBCs was observed under these conditions in this study. In addition to the stiffening of SCT RBCs, other exercise-induced physiological changes can also possibly lead to a higher risk. For example, higher epinephrine during exercise has an effect on SCT RBCs' adhesion ${ }^{42,43}$. Increased adhesion can contribute to stronger interactions between RBCs and epithelial cells and thus trigger inflammatory pathways. Dehydration, a common condition occurring during exercise, has also been reported to affect $\mathrm{RBCs}^{\prime}$ physical properties ${ }^{44}$. The stiffening of SCT RBCs, inflammation, and dehydration individually and together can be associated with a higher risk among SCT individuals during strenuous exercise.

\section{CONCLUSION}

This study sought to address the question of whether RBCs in SCT individuals become stiffened during strenuous exercise. RBCs from SCT individuals and non-SCT subjects were tested under simulated strenuous exercise conditions (i.e., low oxygen and low $\mathrm{pH}$ ). The results show that RBCs from SCT individuals are inherently stiffer and are sensitive to low $\mathrm{pH}$, which induces a significant stiffness increase in SCT RBCs, implying that the stiffening of RBCs could occur in SCT individuals during strenuous exercise. Furthermore, the experimental results revealed that deoxygenation alone did not cause SCT RBCs to increase their stiffness. However, because low oxygen levels contribute to the lowering of blood $\mathrm{pH}$, the stiffening of SCT RBCs in vivo could result from a combined effect of low oxygen and low $\mathrm{pH}$.

\section{ACKNOWLEDGEMENTS}

Financial support from the Natural Sciences and Engineering Research Council of Canada (NSERC) through a Steacie Fellowship, from Grand Challenges of Canada, and from the Canada Research Chairs Program is acknowledged.

\section{COMPETING INTERESTS}

The authors declare no conflict of interest.

\section{REFERENCES}

1 Harmon KG, Asif IM, Klossner D et al. Incidence of sudden cardiac death in national collegiate athletic association athletes. Circulation 2011; 123: 1594-1600.

2 Maron BJ, Doerer JJ, Haas TS et al. Sudden deaths in young competitive athletes analysis of 1866 deaths in the United States, 1980-2006. Circulation 2009; 119: 1085-1092.

3 Hyslop DM. Sudden death in young athletes. The New England Journal of Medicine 1923; 329: 1738.

4 Harmon KG, Drezner Ja, Klossner D et al. Sickle cell trait associated with a RR of death of 37 times in national collegiate athletic association football athletes: A database with 2 million athlete-years as the denominator. British Journal of Sports Medicine 2012; 46: 325-330.

5 Connes P, Reid H, Hardy-Dessources MD et al. Physiological responses of sickle cell trait carriers during exercise. Sports Medicine 2008; 38: 931-946.

6 Tsaras G, Owusu-Ansah A, Boateng FO et al. Complications associated with sickle cell trait: A brief narrative review. The American Journal of Medicine 2009; 122: $507-512$.

7 Thompson AA. Sickle cell trait testing and athletic participation: A solution in search of a problem? ASH Hematology, The Education Program 2013; 2013: 632-637.

8 Ashley-Koch A, Yang Q, Olney RS. Sickle hemoglobin (Hb S) allele and sickle cell disease: A HuGe review. American Journal of Epidemiology 2000; 160: 825-841.
9 Bunn HF. The triumph of good over evil: Protection by the sickle gene against malaria. Blood 2013; 121: 20-25.

10 Mitchell BL. Sickle cell trait and sudden death--bringing it home. Journal of the National Medical Association 2007; 99: 300-305.

11 Tarini Beth A, Brooks Margaret Alison, Bundy David G. A policy impact analysis of the mandatory NCAA sickle cell trait screening program. Health Services Research 2012; 47: 446-461.

12 Julien Tripette, Dessources Hardy, Dominique Marie et al. Exercise-related complications in sickle cell trait. Clinical Hemorheology and Microcirculation 2013; 55: 29-37.

13 Higgins JM, Eddington DT, Bhatia SN et al. Sickle cell vasoocclusion and rescue in a microfluidic device. Proceedings of the National Academy of Sciences of the United States of America 2007; 104: 20496-20500.

14 Barabino Gilda A, Platt Manu O, Kaul Dhananjay K. Sickle cell biomechanics. Annual Review of Biomedical Engineering 2010; 12: 345-367.

15 Osnes JB, Hermansen L. Acid-base balance after maximal exercise of short duration. Journal of Applied Physiology 1972; 32: 59-63.

16 Hermansen L, Osnes JB. Blood and muscle $\mathrm{pH}$ after maximal exercise in man. Journal of Applied Physiology 1972; 32: 304-308.

17 Tachev KD, Angarska JK, Danov KD et al. Erythrocyte attachment to substrates: Determination of membrane tension and adhesion energy. Colloids and Surfaces B: Biointerfaces 2000; 19: 61-80.

18 Merkel TC, Bondar VI, Nagai K et al. Gas sorption, diffusion, and permeation in poly (dimethylsiloxane). Journal of Polymer Sciences B: Polymer Physics 2000; 38: 415-434.

19 Zheng Yi, Cachia Mark A, Ge Ji et al. Mechanical differences of sickle cell trait (SCT) and normal red blood cells. Lab on a Chip 2015; 15: 3138-3146.

20 Reneman Robert S, Hoeks AP. Wall shear stress as measured in vivo: Consequences for the design of the arterial system. Medical \& Biological Engineering \& Computing 2008; 46: 499-507.

21 Evans EA. New membrane concept applied to the analysis of fluid shear- and micropipette-deformed red blood cells. Biophysical Journal 1973; 13: 941-954.

22 Braunmüller Susanne, Schmid Lothar, Sackmannc Erich et al. Hydrodynamic deformation reveals two coupled modes/time scales of red blood cell relaxation. Soft Matter 2012; 8: 11240-11248.

23 Evans EA, Waugh R, Melnik L. Elastic area compressibility modulus of red cell membrane. Biophysical Journal 1976; 16: 585-595.

24 Liu Xiao, Tang Zhi-yu, Zeng Zhu et al. The measurement of shear modulus and membrane surface viscosity of RBC membrane with Ektacytometry: A new technique. Mathematical Biosciences 2007; 209: 190-204.

25 Maciaszek Jamie L, Lykotrafitis George. Sickle cell trait human erythrocytes are significantly stiffer than normal. Journal of Biomechanics 2011; 44: 657-661.

26 Schauf B, Lang U, Stute P et al. Reduced red blood cell deformability, an indicator for high fetal or maternal risk, is found in preeclampsia and IUGR. Hypertension in Pregnancy 2002; 21: 147-160.

27 Baskurt OK, Meiselman HJ. Blood rheology and hemodynamics. Seminars in Thrombosis and Hemostasis 2003; 29: 435-450.

28 Hakim TS. Erythrocyte deformability and segmental pulmonary vascular resistance: Osmolarity and heat treatment. Journal of Applied Physiology 1988; 65: 1634-1641.

29 Vincent Lucile, Féasson Léonard, Oyono-Enguéllé Samuel et al. Remodeling of skeletal muscle microvasculature in sickle cell trait and alpha-thalassemia. American Journal of Physiology: Heart and Circulatory Physiology 2010; 298: $\mathrm{H} 375-\mathrm{H} 384$.

30 Kuzman D, Žnidarčič T, Gros $\mathrm{M}$ et al. Effect of $\mathrm{pH}$ on red blood cell deformability. Pflügers Archiv 2000; 440: 193-194.

31 Simmonds Michael J, Connes Philippe, Sabapathy Surendran. Exercise-induced blood lactate increase does not change red blood cell deformability in cyclists. PLoS One 2013; 8: e71219.

32 Norbert Nemeth, Iren Miko, Andrea Furka et al. Concerning the importance of changes in hemorheological parameters caused by acid-base and blood gas alterations in experimental surgical models. Clinical Hemorheology and Microcirculation 2012; 51: 43-50.

33 Philippe Connes, Olivier Hue, Julien Tripette et al. Blood rheology abnormalities and vascular cell adhesion mechanisms in sickle cell trait carriers during exercise. Clinical Hemorheology and Microcirculation 2008; 39: 179-184.

34 Schreier David A, Forouzan Omid, Hacker Timothy A et al. Increased red blood cell stiffness increases pulmonary vascular resistance and pulmonary arterial pressure. Journal of Biomechanical Engineering 2016; 138: 021012.

35 Campos Jessica, Lobo Clarisse, Queiroz Ana Maria Mach et al. Treatment of the acute sickle cell vaso-occlusive crisis in the emergency department: A Brazilian method of switching from intravenous to oral morphine. European Journal of Haematology 2014; 93: 34-40. 
36 Liu Fei, Mizukami Hiroshi, Sarnaik Sharada et al. Calcium-dependent human erythrocyte cytoskeleton stability analysis through atomic force microscopy. Journal of Structural Biology 2005; 150: 200-210.

37 Low PS. Structure and function of the cytoplasmic domain of band 3: Center of erythrocyte membrane-peripheral protein interactions. Biochimica et Biophysica Acta-Reviews on Biomembranes 1986; 864: 145-167.

38 Fagnété Sara, Philippe Connes, Olivier Hue et al. Faster lactate transport across red blood cell membrane in sickle cell trait carriers. Journal of Applied Physiology 2006; 100: 427-432.

39 Wahl Miriam L, Owen Judith A, Burd Randy et al. Regulation of intracellular pH in human melanoma: Potential therapeutic implications. Molecular Cancer Therapeutics 2002; 1: 617-628.

40 Connes Philippe, Sara Fagnété, Hardy-Dessources Marie-Dominique et al. Does higher red blood cell (RBC) lactate transporter activity explain impaired RBC deformability in sickle cell trait? The Japanese Journal of Physiology 2005; 55: 385-387.

41 Robergs Robert A, Ghiasvand Farzenah, Parker Daryl. Biochemistry of exerciseinduced metabolic acidosis. American Journal of Physology-Regulatory, Integrative and Comparative Physiology 2004; 287: R502-R516.
42 Maciaszek Jamie L, Andemariam Biree, Huber Greg et al. Epinephrine modulates BCAM/Lu and ICAM-4 expression on the sickle cell trait red blood cell membrane. Biophysical Journal 2012; 102: 1137-1143.

43 Aubron Cécile, Nichol Alistair, Cooper D Jamie et al. Age of red blood cells and transfusion in critically ill patients. Annals of Intensive Care 2013; 3: 2.

44 Nitin John A. Review of clinical profile in sickle cell traits. Oman Medical Journal 2010; 25: 3-8.

(c) This work is licensed under a Creative Commons Attribution 4.0 article are included in the article's Creative Commons license, unless indicated otherwise in the credit line; if the material is not included under the Creative Commons license, users will need to obtain permission from the license holder to reproduce the material. To view a copy of this license, visit http://creativecommons.org/licenses/ by/4.0/

(c) The Author(s) 2016 